\title{
Advances in the Treatment of Metastatic Colorectal Cancer
}

\author{
C Kurkjian ${ }^{1}$ and $\mathbf{S}$ Kummar ${ }^{2}$ \\ ${ }^{1}$ Division of Cancer Treatment and Diagnosis, National Cancer Institute, Bethesda, MD 20892 \\ ${ }^{2}$ Medial Oncology Branch, Center for Clinical Research, National Cancer Institute, Bethesda, MD \\ 20892
}

\begin{abstract}
The treatment of metastatic colorectal cancer has undergone major advances yielding significant improvements in survival over the past decade. These advances have evolved due to the benefits of combination chemotherapy as well as the incorporation of biologic therapy. However, as we struggle to provide optimum care while sparing patients ineffective therapy and undue cost, the importance of tailored therapy to maximize benefit will become increasingly important. This article reviews the major advances in the treatment of patients with metastatic colorectal cancer as well as the burgeoning developments in individualized therapy.
\end{abstract}

\section{Introduction}

Despite improvements in screening programs for colorectal cancer approximately $20 \%$ of patients continue to present with metastatic disease.(1) Furthermore, in a pooled analysis of the results from seven randomized trials in patients with stage II or III colorectal cancer who underwent either surgery alone or surgery followed by adjuvant 5-fluorouracil (FU) with either levamisole or leucovorin, the disease free survival at 5 years was $67 \%$ in the patients who received chemotherapy and 55\% in those who only underwent surgery highlighting the fact that a proportion of patients suffer recurrence despite adjuvant treatment.(2) While the addition of oxaliplatin to adjuvant 5-FU regimens has resulted in 3-year disease free survival rates of $76 \%$ to $78 \%$, recurrent disease is still encountered in many patients such that a review of novel approaches in the treatment of metastatic disease is pertinent. $(3,4)$ The treatment of carcinoma of the colon and rectum has seen great strides with a doubling of median overall survival in the last decade.(5-9) This has been most evident with the widespread incorporation of biologic agents into modern therapeutic approaches. The struggle to improve therapies in metastatic disease has provided the landscape for the adoption of novel biologic agents into treatment regimens in the recent past and will continue to serve in this capacity as we move into the next era of individualized therapeutic approaches.

\section{Evolution of Chemotherapeutic Regimens in Metastatic Disease}

\section{5-Fluorouracil}

Much has changed since the initial Food and Drug Administration (FDA) approval of 5FU in 1962 (http://www.accessdata.fda.gov). FU, an analogue of uracil, has activity in cancer cells

Corresponding Author: Dr Shivaani Kummar, Medical Oncology Branch, National Cancer Institute, 10 Center Drive, 10/12N226, Bethesda, MD 20892, Tel: (301)435-5402, Fax: (301)402-0172, E-mail: kummars@ mail.nih.gov.

Publisher's Disclaimer: Disclaimer: "The content of this publication does not necessarily reflect the views or policies of the Department of Health and Human Services, nor does mention of trade names, commercial products, or organizations imply endorsement by the U.S. Government." 
through its conversion to several active metabolites which result in the disruption of RNA synthesis as well as inhibition of the rate-limiting enzyme in nucleotide synthesis, thymidylate synthase (TS).(10) Because the activity of single agent 5-FU as first line therapy was in the range of 10-20\%(11), novel ways to increase its efficacy were actively sought. The addition of leucovorin improved FU activity by increasing the availability of 5,10-methylene tetrahydrofolate which then enhanced the inhibition of TS by the FU metabolite, fluorodeoxyuridine monophosphate (FdUMP).(10) Levamisole, an anti-helminthic drug with in vitro anti-cancer activity, was also combined with $\mathrm{FU}$ in patients with advanced colorectal cancer; however, efficacy with the addition of levamisole was less than that with the addition of leucovorin.(12,13) Further modulation of FU therapy came with investigations into the efficacy and toxicity of bolus regimens compared to infusional administration. More definitive results in the comparison of the two modes of administration came with a meta-analysis of over 1200 patients from 6 randomized trials demonstrating that continuous infusion 5-FU was associated with a significantly higher response rate $(22 \% \mathrm{v} 14 \%$, overall response odds ratio of $0.55 ; 95$ percent confidence interval $=0.41$ to $0.75, \mathrm{p}=0.0002$ ) as well as increased overall survival.(14) Toxicities differed between the two regimens with the bolus regimen resulting in more hematologic toxicity and the infusional schedule causing more cases of hand-foot syndrome. Thus was established the infusional 5-FU backbone that would become an integral part of the chemotherapeutic regimens in use today.

\section{Capecitabine}

Due to its rapid degradation and inconsistent gastrointestinal absorption, FU can only be administered intravenously. These inadequacies led to the search for FU prodrugs which could be taken orally. Capecitabine, a third generation oral fluoropyrimidine, was approved by the FDA in 2001 for the treatment of advanced colorectal cancer. Three enzymatic steps result in the intratumoral formation of FU from capecitabine, the last step catalyzed by thymidine phosphorylase which is preferentially found within tumor tissue.(15) In an analysis of the two randomized phase III trials comparing the administration of capecitabine with a 4-weekly bolus FU/leucovorin regimen in over 1200 patients with advanced disease, the response rate in the oral therapy group was higher than the bolus regimen $(26 \% \mathrm{v} 17 \%, \mathrm{p}<0.0002) .(16)$ Overall survival rates were similar between the two groups. While the overall toxicity profile favored the capecitabine treated groups, the incidences of hand-foot syndrome and hyperbilirubinemia were greater in the patients treated with the oral agent.

\section{Combination Chemotherapy}

\section{Irinotecan}

To further the gains made by optimal modulation of FU therapy, additional chemotherapeutic agents were investigated in combination with an FU backbone leading to the regimens widely used today. Based on the activity of single agent irinotecan, a topoisomerase I inhibitor, after fluorouracil failure in randomized controlled trials comparing the agent to supportive care (17) and to infusional FU therapy(18), respectively, the combination of irinotecan and FU/ leuocovorin therapy was tested. In a randomized trial of nearly 700 patients with advanced colorectal cancer, irinotecan in combination with bolus FU/leucovorin was compared with irinotecan alone and FU/leuocovorin alone.(19) The overall survival for the patients who received all three agents was significantly longer than for those who received only FU/ leuocovorin $(14.8$ months vs. 12.6 months, $\mathrm{p}=0.04)$. For the patients who were treated with irinotecan alone, the survival was not significantly different than in those who were treated with FU/leuocovorin. Though the incidence of grade 3 diarrhea was significantly higher in the irinotecan treated groups, the incidence of grade 4 diarrhea was the same among all patients groups. Irinotecan was also investigated in combination with infusional $F U(20)$ and the overall survival was 17.4 months in the combination therapy group compared to 14.1 months in the 
group receiving infusional FU/leucovorin alone ( $\mathrm{p}=0.031$ ). Although grade 3 and 4 toxicities were greater in the combination therapy group, these were reversible and easily managed.

\section{Oxaliplatin}

Phase II trials of oxaliplatin alone demonstrated response rates of approximately $10 \%$ in patients with advanced colorectal cancer whose disease has progressed on FU therapy.(21, 22) However, its additive effects in vitro and in vivo when used in combination with FU prompted clinical trials of the combination and demonstrated response rates of $20 \%$ to $50 \%$. (23-25) In a pivotal phase III trial, FU given as a bolus and then continuous infusion was administered with or without oxaliplatin every 2 weeks.(26) Determination of median progression free survival in months was the primary objective, and the addition of oxaliplatin imparted a significant increase in the duration of this endpoint (9.0 v. 6.2 months, $p=0.0003$ ). While there was an improvement in overall survival, it was not statistically significant. The addition of oxaliplatin did result in higher rates of grade 3 and/or 4 toxicity in the categories of neutropenia, peripheral neurotoxicity and diarrhea. Thus, the combination of FU bolus/ infusion and oxaliplatin (also termed FOLFOX) was added to the arsenal of therapies for advanced disease. The initial trials of FOLFOX utilized an oxaliplatin dose of $85 \mathrm{mg} / \mathrm{m}^{2}$. Modification of the oxaliplatin dosing as well as the amount of FU administered as a bolus would be subsequently undertaken in a variety of trials. Table 1 outlines the dosing and schedule of the various modifications of the FOLFOX regimen.

\section{Irinotecan vs. Oxaliplatin}

With the respective addition of two chemotherapeutic agents to the well-accepted FU/ leuocovorin backbone resulting in never before seen efficacy in metastatic colorectal cancer came the question of whether one combination was superior to the other. In the phase III Intergroup study, N9741, Goldberg and colleagues compared the efficacy of three different regimens: irinotecan in combination with bolus FU/leucovorin, known as IFL, irinotecan and oxaliplatin given on day 1 every 3 weeks, known as IROX, and FOLFOX 4.(6) The median time to progression of 8.7 months, response rate of $45 \%$ and median survival of 19.5 months all favored the FOLFOX arm over both other arms. The patients who received FOLFOX also had lower rates of severe nausea and vomiting, diarrhea and febrile neutropenia, although patients who received either of the oxaliplatin containing regimens suffered more sensory neuropathy and neutropenia. Thus, the superiority of oxaliplatin with FU was established over bolus FU with irinotecan. Interestingly, $60 \%$ of patients who initially received FOLFOX then went on to receive irinotecan containing therapy whereas only $24 \%$ of those patients who initially received IFL subsequently received oxaliplatin as second-line therapy. This inequity was due to drug supply and the lack of wide-spread availability of oxaliplatin in the US. Subsequently, the question arose of whether the improved survival noted for the FOLFOX arm could be attributed to the utilization of irinotecan upon progression. The results implied that exposure to all 3 drugs (FU, oxaliplatin and irinotecan) could result in median survivals in the range of 19-20 months. However, the question of which regimen to use and in what sequence to administer remained. Tournigand, et al. addressed this issue in a trial in which half the patients with chemo-naïve metastatic colorectal cancer were randomized to receive FOLFOX6 and the other half received FOLFIRI.(27) Approximately 110 patients were randomized to each arm and median survival in the patients who received FOLFOX first followed by FOLFIRI was 20.6 months compared with 21.5 months in the patients who received FOLFIRI first followed by FOLFOX ( $\mathrm{p}=0.99)$. This lack of difference in survival existed despite a higher response rate in second line FOLFOX at $15 \%$ compared with $4 \%$ in the patients who received FOLFIRI. Median PFS in first line therapy was not statistically different between the two arms. There was a higher incidence of alopecia, mucositis, and nausea/vomiting in the patients who were treated with first-line FOLFIRI with increased rates of neutropenia and neurosensory toxicity in those who initially received FOLFOX. The findings of this critical trial as well as 
the results from an analysis by Grothey, et al. of seven randomized phase III trials in advanced colorectal cancer established that regardless of the sequence of treatment, patient survival was improved by exposure to all three active drugs.(28)

\section{Intermittent vs. Continuous Therapy}

The addition of oxaliplatin to FU/LV certainly changed the standard of care in patients with advanced colorectal cancer; however, its cumulative neurotoxicity often required treatment interruption despite continued benefit. This prompted the inquiry into whether patients could undergo a more tolerable intermittent therapy without sacrificing efficacy. In the OPTIMOX1 trial, over 600 patients were randomized to receive either FOLFOX4 every 2 weeks continuously until progression or FOLFOX7 for 6 cycles, followed by FU/LV alone for 12 cycles with subsequent reintroduction of FOLFOX7.(29) While there was no difference in progression-free survival or overall survival between the two treatment approaches, only $40.1 \%$ of patients went on to undergo reintroduction of oxaliplatin. Neurotoxicity rates did not differ significantly between the two arms. While it appeared that oxaliplatin could be safely stopped after 6 cycles, it is unclear if a more consistent reintroduction of oxaliplatin could have elicited improved benefit. The OPTIMOX 2 study took this strategy further with a complete cessation of therapy in a randomized phase II trial comparing FOLFOX7 for 6 cycles followed by either a chemotherapy free interval followed by reintroduction of FOLFOX7 at the time of tumor progression or 12 cycles of FU/LV before FOLFOX7 reintroduction, the latter identical to the OPTIMOX1 strategy.(30) Approximately 200 patients were enrolled in the trial and results demonstrated that there was a trend toward an inferior overall survival in the patients who were given a chemotherapy free interval, though this did not meet statistical significance (26 months vs. 19 months, $\mathrm{p}=0.05$ ). The median duration of the chemotherapy free interval was 4.6 months in patients who underwent treatment interruption and did not undergo resection of any metastasis. The response rate after reintroduction of oxaliplatin did not differ between the two groups. The OPTIMOX2 trial investigators have taken a similar approach in the ongoing DREAM trial in which biologic therapy alone is continued after initially administering with chemotherapy.

\section{Biologic Therapies}

As the understanding of molecular pathways driving colon carcinogenesis has evolved, the development of targeted therapies has provided further gains on recent advances in chemotherapy approaches in this disease. Though the activity of these agents used alone has not translated into substantial responses, their use in combination with chemotherapy has resulted in improvement in response and survival rates. Three targeted agents have been approved by the FDA for the treatment of metastatic colorectal cancer including bevacizumab, cetuximab, and panitumumab. The results of several of the key trials which have led to current chemotherapeutic and biologic treatments of patients with metastatic disease are included in Table 2.

\section{Individualized Therapy}

While the advent of biologic therapies and newer chemotherapy combinations as well as the efficacy of selected surgical resection in patients with metastatic disease has yielded significant gains, metastatic colorectal cancer remains a largely incurable disease. Furthermore, these novel treatments have come with a price, due to monetary costs and treatment related adverse outcomes. Out of this has arisen the growing tide of individualized therapies aimed at providing the optimum treatment based on pharmacogenomic and tumor specific analyses. The following is a brief discussion of some of the advances made in this arena. 


\section{Irinotecan Metabolism}

The active metabolite of irinotecan, $\mathrm{SN}-38$, undergoes glucuronidation to an inactive form by the enzyme uridine diphosphate glucuronosyl transferase (UGT1A1).(31) Expression of the enzyme in the liver is not uniform among patients and the amount of UGT1A1 produced depends on the number of T-A repeats in the promoter region of its gene. The wild-type of the enzyme has 6 T-A repeats whereas the presence of 7 repeats results in diminished UGT1A1 levels, the latter causing increased levels of SN-38 after irinotecan exposure. Patients who are homozygous for the $\mathrm{TA}_{7}$ allele (also known as Gilbert's syndrome, a common cause of mild indirect hyperbilirubinemia) have been shown to be more susceptible to the toxic effects of SN-38, namely neutropenia and diarrhea, as evidenced in multiple studies.(32-34) These findings led to the FDA approving a change in drug labeling in 2005 to reflect the need for a reduced dose of irinotecan in those patients who are homozygous for the UGT1A1-28* allele, though guidelines for the amount of dose reduction are sparse. Because the activity of irinotecan is dose-dependent, the question of whether patients who are homozygous for the TA6 allele require higher doses of irinotecan for improved activity was addressed.(35) Patients enrolled on the Dutch CAIRO study were randomized to either Arm A (capecitabine alone in first-line, then irinotecan alone in second-line then capecitabine with oxaliplatin in third line treatment) or Arm B (capecitabine with irinotecan in first line (CAPIRI) followed by capecitabine and oxaliplatin). Blood samples from these patients were also obtained to analyze for the UGT1A1 genotype. Though patients with the homozygous $\mathrm{TA}_{7}$ genotype were more susceptible to febrile neutropenia in combination and single agent irinotecan treatment groups (18.2\% vs. $\mathrm{TA}_{6} / \mathrm{TA}_{6} ; 1.5 \%$ and $\mathrm{TA}_{6} / \mathrm{TA}_{7} ; 6.5 \%, \mathrm{p}=0.031$ in the combination group), the dose intensity and number of cycles received did not differ. There was no statistically significant difference in response rates among the various genotypes. Similarly, diarrhea rates did not differ for patients in the various groups. The results of the Dutch Colorectal Cancer Group Study demonstrated tolerability for full doses of irinotecan among those patients with homozygous $\mathrm{TA}_{7}$ genotype, somewhat contradictory to other studies. However, this was the largest series to date and included over 250 patients from the CAIRO study in whom DNA was obtained. Thus, in those patients who have a deficiency in expression of UGT1A1, clinicians must at the very least be vigilant in the surveillance of toxic effects when utilizing irinotecan. Similarly, for the majority of patients who have the $\mathrm{TA}_{6} / \mathrm{TA}_{6}$ genotype, it does not appear from this study that any additional benefit is derived from an increased dose of the agent.

\section{Fluoropyrimidine metabolism}

The enzyme dihydropyrimidine dehydrogenase (DPD) is responsible for pyrimidine metabolism and its deficiency has been implicated in severe toxicity after exposure to FU. $(36,37)$ Based on pharmacokinetic variability due to differing DPD levels, some patients experience benefit and tolerate the drug without issue whereas others experience no response at all or suffer from severe toxic effects. $(36,37)$ Deficiency in DPD activity has been shown to have a genetic predisposition and approximately $0.1 \%$ of the population has a complete deficiency with partial deficiency detected in 3-5\%.(14) The pharmacokinetic variability among individuals results in a variability in toxicity and efficacy as evidenced by the finding that responses can be improved with maintenance of higher FU area under the curve (AUC) levels. $(37,38)$ In prospective trials, Gamelin and colleagues demonstrated that an $\mathrm{AUC}_{0-8}$ of 20 to $25 \mathrm{mg} \cdot \mathrm{h} \cdot \mathrm{L}^{-1}$ was the level at which FU activity was optimized and toxicity minimized. $(36,37)$ In these studies, FU was utilized as a continuous infusion for 8 hours every week. Based on the encouraging results of these preliminary studies, a multicenter, randomized phase III trial investigating a pharmacokinetically guided dosing of FU with high dose leucovorin was undertaken in patients with advanced colorectal cancer.(39) Patients were randomized to either FU dosing based on body surface area at $1500 \mathrm{mg} / \mathrm{m}^{2} /$ week administered as an 8 hour infusion (arm A) or FU at an initial dose of $1500 \mathrm{mg} / \mathrm{m}^{2} /$ week over 8 hours which was subsequently adjusted based on pharmacokinetic measurements from cycle 2 onward targeting an AUC of 
20-25 mg*hr/L (arm B). Leuocovorin dosing remained at $200 \mathrm{mg} / \mathrm{m} 2 /$ week in arms A and B. Over 200 patients were randomized and an intention to treat analysis demonstrated an overall response rate favoring arm $\mathrm{B}(18.3 \%$ vs. $33.7 \%, \mathrm{p}=0.004)$. While overall survival also favored the phamacokinetically dosed arm, this did not reach statistical significance (16 months vs. 22 months, $\mathrm{p}=0.08)$. Toxicity was greater in arm A than $\mathrm{B}(\mathrm{p}=0.003)$. The survival advantage in arm $B$ is meaningful particularly in light of the 22 month survival with administration of FU as a single agent; however, these results are difficult to interpret in the context of modern, multi-agent regimens. The pharmacokinetic based dose adjustments were performed on samples at hours 3 and 7 of the continuous infusion in this study. Whether this burden of pharmacokinetic dose adjustment can be widely applicable in the clinical setting remains to be determined.

\section{Predicting Efficacy of EGFR Targeting Agents Epidermal Growth Factor Receptor (EGFR)}

Though the addition of monoclonal antibodies to the treatment approach in patients with advanced colorectal cancer has resulted in important survival gains, these advances have come with a significant economic impact. For example, the cost of treating patients with advanced colorectal cancer with irinotecan and cetuximab amounts to approximately thirty thousand dollars for an 8 week course.(40) This staggering expense combined with the fact that a proportion of patients do not derive benefit from cetuximab (or panitumumab) treatment has strengthened the resolve to better predict those patients whose disease will respond to these agents. Based on the mechanism of action of cetuximab and panitumumab, tumor expression of the EGFR by immunohisochemistry (IHC) has been a requirement of research participation in most trials. Additionally, the FDA approval of cetuximab in 2004 and panitumumab in 2006 was only for the treatment of patients with advanced colorectal cancer whose tumors express EGFR. However, evidence suggests no difference in benefit for those patients whose tumors express low levels of EGFR compared with those who have high levels of the receptor.(41, 42) Even patients with negative IHC staining for EGFR seem to demonstrate a response to the agent.(43) The evidence for a benefit in those patients with weak or absent IHC staining for EGFR was in part attributed to difficulties with IHC testing such as its inherent variability in results. Subsequently, investigations into a correlation between cetuximab or panitumumab response and an increase in gene copy number by fluorescent in situ hybridization (FISH) were undertaken. In one of the first studies published, Moroni and colleagues tested 31 samples of patients treated with cetuximab or panitumumab for genetic changes in EGFR by FISH.(44) Of the nine patients who responded to the anti-EGFR therapy, eight had an increase in the EGFR copy number whereas only 1 of 21 non-responders demonstrated such genetic changes. In contrast, subsequent studies have not found such a strong correlation between increased copy number and response to cetuximab or panitumumab.(45-47) Thus, the conclusions drawn from these investigations suggest that a high EGFR gene copy number does not necessarily predict success with EGFR targeted therapy; and, perhaps more importantly, a low copy number likely portends a lack of benefit. While the adoption of these results into routine practice would appear to save patients from ineffective treatment, the inability to reliably define EGFR patterns as well as a variability in results have proven to be major hurdles in the routine applicability of the practice.(48)

\section{Relationship between KRAS status and response to EGFR targeted therapy}

Stimulation of the EGFR at the cell surface has several downstream effects, including activation of the signal transducer, KRAS.(49,50) In approximately $30 \%$ to $50 \%$ of colorectal cancers, KRAS can be constitutively activated such that inhibition of EGFR signaling will have no effect on KRAS activity resulting in continued cancer cell growth.(49-54) One of the pivotal studies demonstrating a lack of response to EGFR inhibition in colorectal cancer patients with 
a mutated KRAS was performed in a retrospective analysis of the phase III randomized study comparing panitumumab to best supportive care (BSC).(55) When comparing the effect of panitumumab vs. BSC on progression free survival in patients with wild-type KRAS, the treatment effect was much greater (HR $0.45 ; 95 \%$ confidence interval, 0.34 to 0.59 ; median PFS in panitumumab was 12.3 weeks and in BSC was 7.3 weeks) than in patients with mutated KRAS (HR, 0.99; 95\% confidence interval, 0.73 to 1.36; median PFS in panitumumab was 7.4 weeks and in BSC was 7.3 weeks). Based on the results of this study, the European Committee for Medicinal Products for Human Use made the decision to require confirmation of wild-type KRAS status prior to initiation of panitumumab therapy.(56) Multiple retrospective studies have demonstrated similar results with a lack of response in KRAS mutant tumors. The most recent studies presented at the American Society of Clinical Oncology Annual Meeting in 2008 are outlined in Table 3. While no prospective trial has been performed corroborating these retrospective findings, the consistency of results in the multiple analyses carried out to date will likely change practice patterns. Unlike European authorities, no federal regulatory action has yet been taken to require KRAS testing prior to cetuximab or panitumumab therapy in the US.

\section{Conclusions}

The landscape of therapy in metastatic colorectal cancer has changed remarkably in the last decade. The routine utilization of combination chemotherapy with biological therapies has afforded patients unprecedented overall survival rates. However, these advances have come with a price, both in terms of cost as well as toxicity. The continued evolution of personalized therapy will hopefully maximize therapeutic gains while minimizing any undue burden.

Oncologists have been forced to accept the risks associated with administering a treatment that may provide no gains to the patient in an effort to avoid denial of treatment to those who may benefit. The recent results suggesting a lack of response to EGFR modulating therapy in patients with a mutated KRAS gene is the first of hopefully many more advances to come in the arena of individualized treatment. The ever-increasing challenge of developing comprehensive therapy, too often cost-prohibitive and without universal efficacy, has encouraged the research community to expedite the search for biomarkers truly predictive of therapeutic response.

\section{Acknowledgments}

This research was supported in part by the Intramural Research Program of the NIH, National Cancer Institute, Center for Cancer Research, Bethesda, MD.

\section{References}

1. Jemal A, Siegel R, Ward E, et al. Cancer Statistics, 2008. CA Cancer J Clin 2008;58:71-96. [PubMed: 18287387]

2. Gill S, Loprinzi CL, Sargent DJ, et al. Pooled Analysis of Fluorouracil-Based Adjuvant Therapy for Stage II and III Colon Cancer: Who Benefits and by How Much? J Clin Oncol 2004;22:1797-806. [PubMed: 15067028]

3. Andre T, Boni C, Mounedji-Boudiaf L, et al. Oxaliplatin, Fluorouracil, and Leucovorin as Adjuvant Treatment for Colon Cancer. N Engl J Med 2004;350:2343-51. [PubMed: 15175436]

4. Kuebler JP, Wieand HS, O'Connell MJ, et al. Oxaliplatin Combined With Weekly Bolus Fluorouracil and Leucovorin As Surgical Adjuvant Chemotherapy for Stage II and III Colon Cancer: Results From NSABP C-07. J Clin Oncol 2007;25:2198-204. [PubMed: 17470851]

5. Budd GT, Fleming TR, Bukowski RM, et al. 5-Fluorouracil and folinic acid in the treatment of metastatic colorectal cancer: a randomized comparison. A Southwest Oncology Group Study. J Clin Oncol 1987;5:272-7. [PubMed: 3543246] 
6. Goldberg RM, Sargent DJ, Morton RF, et al. A Randomized Controlled Trial of Fluorouracil Plus Leucovorin, Irinotecan, and Oxaliplatin Combinations in Patients With Previously Untreated Metastatic Colorectal Cancer. J Clin Oncol 2004;22:23-30. [PubMed: 14665611]

7. Hurwitz H, Fehrenbacher L, Novotny W, et al. Bevacizumab plus Irinotecan, Fluorouracil, and Leucovorin for Metastatic Colorectal Cancer. N Engl J Med 2004;350:2335-42. [PubMed: 15175435]

8. Kabbinavar FF, Hambleton J, Mass RD, Hurwitz HI, Bergsland E, Sarkar S. Combined Analysis of Efficacy: The Addition of Bevacizumab to Fluorouracil/Leucovorin Improves Survival for Patients With Metastatic Colorectal Cancer. J Clin Oncol 2005;23:3706-12. [PubMed: 15867200]

9. The Meta-Analysis Group in C. Modulation of Fluorouracil by Leucovorin in Patients With Advanced Colorectal Cancer: An Updated Meta-Analysis. J Clin Oncol 2004;22:3766-75. [PubMed: 15365073]

10. Longley DB, Harkin DP, Johnston PG. 5-Fluorouracil: mechanisms of action and clinical strategies. Nat Rev Cancer 2003;3:330-8. [PubMed: 12724731]

11. Johnston PG, Kaye S. Capecitabine: a novel agent for the treatment of solid tumors. Anticancer Drugs 2001;12:639-46. [PubMed: 11604550]

12. Porschen R, Bermann A, Loffler T, et al. Fluorouracil Plus Leucovorin as Effective Adjuvant Chemotherapy in Curatively Resected Stage III Colon Cancer: Results of the Trial adjCCA-01. J Clin Oncol 2001;19:1787-94. [PubMed: 11251010]

13. Wolmark N, Rockette H, Mamounas E, et al. Clinical Trial to Assess the Relative Efficacy of Fluorouracil and Leucovorin, Fluorouracil and Levamisole, and Fluorouracil, Leucovorin, and Levamisole in Patients With Dukes' B and C Carcinoma of the Colon: Results From National Surgical Adjuvant Breast and Bowel Project C-04. J Clin Oncol 1999;17:3553-9. [PubMed: 10550154]

14. Meta-analysis Group In Cancer. Efficacy of intravenous continuous infusion of fluorouracil compared with bolus administration in advanced colorectal cancer. J Clin Oncol 1998;16:301-8. [PubMed: 9440757]

15. Walko CM, Lindley C. Capecitabine: A review. Clinical Therapeutics 2005;27:23-44. [PubMed: 15763604]

16. Van Cutsem E, Hoff PM, Harper P, et al. Oral capecitabine vs intravenous 5-fluorouracil and leucovorin: integrated efficacy data and novel analyses from two large, randomised, phase III trials. Br J Cancer 2004;90:1190-7. [PubMed: 15026800]

17. Cunningham D, Pyrhönen S, James RD, et al. Randomised trial of irinotecan plus supportive care versus supportive care alone after fluorouracil failure for patients with metastatic colorectal cancer. The Lancet 1998;352:1413-8.

18. Rougier P, Van Cutsem E, Bajetta E, et al. Randomised trial of irinotecan versus fluorouracil by continuous infusion after fluorouracil failure in patients with metastatic colorectal cancer. The Lancet 1998;352:1407-12.

19. Saltz LB, Cox JV, Blanke C, et al. Irinotecan plus Fluorouracil and Leucovorin for Metastatic Colorectal Cancer. N Engl J Med 2000;343:905-14. [PubMed: 11006366]

20. Douillard JY, Cunningham D, Roth AD, et al. Irinotecan combined with fluorouracil compared with fluorouracil alone as first-line treatment for metastatic colorectal cancer: a multicentre randomised trial. The Lancet 2000;355:1041-7.

21. Levi F, Perpoint B, Garufi C, et al. Oxaliplatin activity against metastatic colorectal cancer. A phase II study of 5-day continuous venous infusion at circadian rhythm modulated rate. Eur J Cancer 1993;29A:1280-4. [PubMed: 8343268]

22. Machover D, Diaz-Rubio E, de Gramont A, et al. Two consecutive phase II studies of oxaliplatin (LOHP) for treatment of patients with advanced colorectal carcinoma who were resistant to previous treatment with fluoropyrimidines. Ann Oncol 1996;7:95-8. [PubMed: 9081400]

23. Andre T, Louvet C, Raymond E, Tournigand C, de Gramont A. Bimonthly high-dose leucovorin, 5fluorouracil infusion and oxaliplatin (FOLFOX3) for metastatic colorectal cancer resistant to the same leucovorin and 5-fluorouracil regimen. Ann Oncol 1998;9:1251-3. [PubMed: 9862058]

24. de Gramont A, Vignoud J, Tournigand C, et al. Oxaliplatin with high-dose leucovorin and 5fluorouracil 48-hour continuous infusion in pretreated metastatic colorectal cancer. Eur J Cancer 1997;33:214-9. [PubMed: 9135491]

25. Levi FA, Zidani R, Vannetzel J-M, et al. Chronomodulated Versus Fixed-Infusion--Rate Delivery of Ambulatory Chemotherapy With Oxaliplatin, Fluorouracil, and Folinic Acid (Leucovorin) in Patients 
With Colorectal Cancer Metastases: a Randomized Multi-institutional Trial. J Natl Cancer Inst 1994;86:1608-17. [PubMed: 7932825]

26. de Gramont A, Figer A, Seymour M, et al. Leucovorin and Fluorouracil With or Without Oxaliplatin as First-Line Treatment in Advanced Colorectal Cancer. J Clin Oncol 2000;18:2938-47. [PubMed: 10944126]

27. Tournigand C, Andre T, Achille E, et al. FOLFIRI Followed by FOLFOX6 or the Reverse Sequence in Advanced Colorectal Cancer: A Randomized GERCOR Study. J Clin Oncol 2004;22:229-37. [PubMed: 14657227]

28. Grothey A, Sargent D, Goldberg RM, Schmoll H-J. Survival of Patients With Advanced Colorectal Cancer Improves With the Availability of Fluorouracil-Leucovorin, Irinotecan, and Oxaliplatin in the Course of Treatment. J Clin Oncol 2004;22:1209-14. [PubMed: 15051767]

29. Tournigand C, Cervantes A, Figer A, et al. OPTIMOX1: a randomized study of FOLFOX4 or FOLFOX7 with oxaliplatin in a stop-and-Go fashion in advanced colorectal cancer--a GERCOR study. J Clin Oncol 2006;24:394-400. [PubMed: 16421419]

30. Maindrault-Goebel F, Lledo G, Chibaudel B, et al. Final results of OPTIMOX2, a large randomized phase II study of maintenance therapy or chemotherapy-free intervals (CFI) after FOLFOX in patients with metastatic colorectal cancer (MRC): A GERCOR study. J Clin Oncol 2007;25:4013.(Meeting Abstracts)

31. Iyer L, King CD, Whitington PF, et al. Genetic predisposition to the metabolism of irinotecan (CPT-11). Role of uridine diphosphate glucuronosyltransferase isoform 1A1 in the glucuronidation of its active metabolite (SN-38) in human liver microsomes. J Clin Invest 1998;101:847-54. [PubMed: 9466980]

32. Innocenti F, Undevia SD, Iyer L, et al. Genetic variants in the UDP-glucuronosyltransferase 1A1 gene predict the risk of severe neutropenia of irinotecan. J Clin Oncol 2004;22:1382-8. [PubMed: 15007088]

33. Iyer L, Das S, Janisch L, et al. UGT1A1*28 polymorphism as a determinant of irinotecan disposition and toxicity. Pharmacogenomics J 2002;2:43-7. [PubMed: 11990381]

34. Marcuello E, Altes A, Menoyo A, Del Rio E, Gomez-Pardo M, Baiget M. UGT1A1 gene variations and irinotecan treatment in patients with metastatic colorectal cancer. Br J Cancer 2004;91:678-82. [PubMed: 15280927]

35. Kweekel DM, Gelderblom H, Van der Straaten T, Antonini NF, Punt CJ, Guchelaar HJ. UGT1A1*28 genotype and irinotecan dosage in patients with metastatic colorectal cancer: a Dutch Colorectal Cancer Group study. Br J Cancer 2008;99:275-82. [PubMed: 18594531]

36. Gamelin E, Boisdron-Celle M, Delva R, et al. Long-term weekly treatment of colorectal metastatic cancer with fluorouracil and leucovorin: results of a multicentric prospective trial of fluorouracil dosage optimization by pharmacokinetic monitoring in 152 patients. 1998;16:1470-8.

37. Gamelin EC, Danquechin-Dorval EM, Dumesnil YF, et al. Relationship between 5-fluorouracil (5FU) dose intensity and therapeutic response in patients with advanced colorectal cancer receiving infusional therapy containing 5-FU. Cancer 1996;77:441-51. [PubMed: 8630950]

38. Milano G, Etienne MC, Renee N, et al. Relationship between fluorouracil systemic exposure and tumor response and patient survival. J Clin Oncol 1994;12:1291-5. [PubMed: 8201391]

39. Gamelin E, Delva R, Jacob J, et al. Individual Fluorouracil Dose Adjustment Based on Pharmacokinetic Follow-Up Compared With Conventional Dosage: Results of a Multicenter Randomized Trial of Patients With Metastatic Colorectal Cancer. J Clin Oncol 2008;26:2099-105. [PubMed: 18445839]

40. Schrag D. The Price Tag on Progress -- Chemotherapy for Colorectal Cancer. N Engl J Med 2004;351:317-9. [PubMed: 15269308]

41. Cunningham D, Humblet Y, Siena S, et al. Cetuximab Monotherapy and Cetuximab plus Irinotecan in Irinotecan-Refractory Metastatic Colorectal Cancer. N Engl J Med 2004;351:337-45. [PubMed: 15269313]

42. Saltz LB, Meropol NJ, Loehrer PJ Sr, Needle MN, Kopit J, Mayer RJ. Phase II Trial of Cetuximab in Patients With Refractory Colorectal Cancer That Expresses the Epidermal Growth Factor Receptor. J Clin Oncol 2004;22:1201-8. [PubMed: 14993230] 
43. Chung KY, Shia J, Kemeny NE, et al. Cetuximab Shows Activity in Colorectal Cancer Patients With Tumors That Do Not Express the Epidermal Growth Factor Receptor by Immunohistochemistry. J Clin Oncol 2005;23:1803-10. [PubMed: 15677699]

44. Moroni M, Veronese S, Benvenuti S, et al. Gene copy number for epidermal growth factor receptor (EGFR) and clinical response to antiEGFR treatment in colorectal cancer: a cohort study. Lancet Oncol 2005;6:279-86. [PubMed: 15863375]

45. Cappuzzo F, Finocchiaro G, Rossi E, et al. EGFR FISH assay predicts for response to cetuximab in chemotherapy refractory colorectal cancer patients. Ann Oncol 2008;19:717-23. [PubMed: 17974556]

46. Frattini M, Saletti P, Romagnani E, et al. PTEN loss of expression predicts cetuximab efficacy in metastatic colorectal cancer patients. Br J Cancer 2007;97:1139-45. [PubMed: 17940504]

47. Sartore-Bianchi A, Moroni M, Veronese S, et al. Epidermal growth factor receptor gene copy number and clinical outcome of metastatic colorectal cancer treated with panitumumab. J Clin Oncol 2007;25:3238-45. [PubMed: 17664472]

48. Moroni M, Sartore-Bianchi A, Veronese S, Siena S. EGFR FISH in colorectal cancer: what is the current reality? Lancet Oncol 2008;9:402-3. [PubMed: 18452847]

49. Downward J. Targeting RAS signalling pathways in cancer therapy. Nat Rev Cancer 2003;3:11-22. [PubMed: 12509763]

50. Schubbert S, Shannon K, Bollag G. Hyperactive Ras in developmental disorders and cancer. Nat Rev Cancer 2007;7:295-308. [PubMed: 17384584]

51. Benvenuti S, Sartore-Bianchi A, Di Nicolantonio F, et al. Oncogenic activation of the RAS/RAF signaling pathway impairs the response of metastatic colorectal cancers to anti-epidermal growth factor receptor antibody therapies. Cancer Res 2007;67:2643-8. [PubMed: 17363584]

52. Bos JL. ras oncogenes in human cancer: a review. Cancer Res 1989;49:4682-9. [PubMed: 2547513]

53. Lievre A, Bachet JB, Le Corre D, et al. KRAS mutation status is predictive of response to cetuximab therapy in colorectal cancer. Cancer Res 2006;66:3992-5. [PubMed: 16618717]

54. Malumbres M, Barbacid M. RAS oncogenes: the first 30 years. Nat Rev Cancer 2003;3:459-65. [PubMed: 12778136]

55. Amado RG, Wolf M, Peeters M, et al. Wild-type KRAS is required for panitumumab efficacy in patients with metastatic colorectal cancer. J Clin Oncol 2008;26:1626-34. [PubMed: 18316791]

56. Baselga J, Rosen N. Determinants of RASistance to anti-epidermal growth factor receptor agents. J Clin Oncol 2008;26:1582-4. [PubMed: 18316790]

57. Cheeseman SL, Joel SP, Chester JD, et al. A /'modified de Gramont/' regimen of fluorouracil, alone and with oxaliplatin, for advanced colorectal cancer. Br J Cancer 87:393-9. [PubMed: 12177775]

58. Hoff PM, Ansari R, Batist G, et al. Comparison of oral capecitabine versus intravenous fluorouracil plus leucovorin as first-line treatment in 605 patients with metastatic colorectal cancer: results of a randomized phase III study. J Clin Oncol 2001;19:2282-92. [PubMed: 11304782]

59. Van Cutsem E, Twelves C, Cassidy J, et al. Oral capecitabine compared with intravenous fluorouracil plus leucovorin in patients with metastatic colorectal cancer: results of a large phase III study. J Clin Oncol 2001;19:4097-106. [PubMed: 11689577]

60. Diaz-Rubio E, Tabernero J, Gomez-Espana A, et al. Phase III study of capecitabine plus oxaliplatin compared with continuous-infusion fluorouracil plus oxaliplatin as first-line therapy in metastatic colorectal cancer: final report of the Spanish Cooperative Group for the Treatment of Digestive Tumors Trial. J Clin Oncol 2007;25:4224-30. [PubMed: 17548839]

61. Fuchs CS, Marshall J, Mitchell E, et al. Randomized, controlled trial of irinotecan plus infusional, bolus, or oral fluoropyrimidines in first-line treatment of metastatic colorectal cancer: results from the BICC-C Study. J Clin Oncol 2007;25:4779-86. [PubMed: 17947725]

62. Giantonio BJ, Catalano PJ, Meropol NJ, et al. Bevacizumab in combination with oxaliplatin, fluorouracil, and leucovorin (FOLFOX4) for previously treated metastatic colorectal cancer: results from the Eastern Cooperative Oncology Group Study E3200. J Clin Oncol 2007;25:1539-44. [PubMed: 17442997]

63. Jonker DJ, O'Callaghan CJ, Karapetis CS, et al. Cetuximab for the treatment of colorectal cancer. N Engl J Med 2007;357:2040-8. [PubMed: 18003960] 
64. Van Cutsem E, Peeters M, Siena S, et al. Open-label phase III trial of panitumumab plus best supportive care compared with best supportive care alone in patients with chemotherapy-refractory metastatic colorectal cancer. J Clin Oncol 2007;25:1658-64. [PubMed: 17470858]

65. Van Cutsem E, Lang I, D'Haens G, et al. KRAS status and efficacy in the first-line treatment of patients with metastatic colorectal cancer (mCRC) treated with FOLFIRI with or without cetuximab: The CRYSTAL experience. J Clin Oncol 2008;26:2.(Meeting Abstracts)

66. Bokemeyer C, Bondarenko I, Hartmann JT, et al. KRAS status and efficacy of first-line treatment of patients with metastatic colorectal cancer (mCRC) with FOLFOX with or without cetuximab: The OPUS experience. J Clin Oncol 2008;26:4000.(Meeting Abstracts)

67. Tejpar S, Peeters M, Humblet Y, et al. Relationship of efficacy with KRAS status (wild type versus mutant) in patients with irinotecan-refractory metastatic colorectal cancer (mCRC), treated with irinotecan (q2w) and escalating doses of cetuximab (q1w): The EVEREST experience (preliminary data). J Clin Oncol 2008;26:4001. [PubMed: 18711191](Meeting Abstracts)

68. Punt CJ, Tol J, Rodenburg CJ, et al. Randomized phase III study of capecitabine, oxaliplatin, and bevacizumab with or without cetuximab in advanced colorectal cancer (ACC), the CAIRO2 study of the Dutch Colorectal Cancer Group (DCCG). J Clin Oncol 2008;26:LBA4011.(Meeting Abstracts) 
Table 1

FOLFOX Regimens

\begin{tabular}{|l|l|l|l|l|}
\hline Regimen & Bolus FU $\left(\mathbf{m g} / \mathbf{m}^{2}\right)$ & Infusional FU $\left(\mathbf{m g} / \mathbf{m}^{2}\right.$ & Oxaliplatin $\left(\mathbf{m g} / \mathbf{m}^{2}\right)$ & Leucovorin $\left(\mathbf{m g} / \mathbf{m}^{2}\right)$ \\
\hline FOLFOX 4(6) & 400 on days 1 and 2 & 600 on days 1 and 2 & 85 & 200 on days 1 and 2 \\
\hline FOLFOX 6(27) & 400 on day 1 & $2400-3000$ & 100 & 400 on day 1 \\
\hline FOLFOX 7(29) & none & 2400 & 130 & 400 on day 1 \\
\hline mFOLFOX6 ${ }^{*}$ (57) & 400 on day 1 & 2400 & 85 & 400 on day 1 \\
\hline
\end{tabular}

* $\mathrm{m}=$ modified 
Table 2

\begin{tabular}{|c|c|c|}
\hline & Regimen & Median Survival in months (p value) \\
\hline Goldberg, et al. 2004(6) & FOLFOX vs. IFL vs. IROX & $\begin{array}{l}19.5 \text { vs. } 15 \text { (0.0001) (FOLFOX vs. IFL) } \\
19.5 \text { vs. } 17.4(0.09) \text { (FOLFOX vs. IROX) } \\
\end{array}$ \\
\hline Tournigand, et al. 2004(27) & FOLFOX $\rightarrow$ FOLFIRI vs. FOLFIRI $\rightarrow$ FOLFOX & 20.6 vs. $21.5(0.99)$ \\
\hline Hoff, et al. 2001(58) & $\begin{array}{l}\text { Capecitabine vs. Bolus } \\
\text { 5FU/leucovorin }\end{array}$ & 12.5 vs. $13.3(0.974)$ \\
\hline Van Cutsem, et al. 2001(59) & $\begin{array}{l}\text { Capecitabine vs. Bolus } \\
\text { 5FU/leucovorin (Mayo Clinic regimen) }\end{array}$ & 13.2 vs $12.1(0.33)$ \\
\hline Diaz-Rubio, et al. 2007(60) & $\begin{array}{l}\text { Capecitabine/oxaliplatin (XELOX) vs. Infusional 5FU/ } \\
\text { oxaliplatin }\end{array}$ & 18.1 vs. $20.8(0.145)$ \\
\hline Fuchs, et al. 2007(61) & $\begin{array}{l}\text { FOLFIRI vs. IFL vs. Capecitabine/Irinotecan (CapeIRI) } \\
\text { (Bevacizumab added after amendment) }\end{array}$ & $\begin{array}{l}\text { FOLFIRI + Bev (not reached) IFL } 19.2(0.007) \\
\text { CapeIRI discontinued due to toxicity related treatment } \\
\text { withdrawal in } 25.5 \%\end{array}$ \\
\hline $\begin{array}{l}\text { Hurwitz, et al. 2004(7) } \\
\text { Giantonio, et al. 2007(62) }\end{array}$ & $\begin{array}{l}\text { IFL vs. IFL + Bevacizumab } \\
\text { FOLFOX4 vs. FOLFOX4 + Bevacizumab }\end{array}$ & $\begin{array}{l}15.6 \text { vs. } 20.3(<0.001) \\
10.9 \text { vs. } 12.8(0.0011)\end{array}$ \\
\hline $\begin{array}{l}\text { Cunningham, et al. 2004(41) } \\
\text { Jonker, et al. 2007(63) }\end{array}$ & $\begin{array}{l}\text { Irinotecan vs. cetuximab + irinotecan } \\
\text { Cetuximab vs. best supportive care (BSC) }\end{array}$ & $\begin{array}{l}1.5 \text { vs. } 4.1(0.48) \\
6.1 \text { vs. } 4.6(0.001) \\
\end{array}$ \\
\hline Van Cutsem, et al. 2007(64) & Panitumumab vs. BSC & Equivalent with $76 \%$ crossover rate from BSC \\
\hline
\end{tabular}


Table 3

\begin{tabular}{|l|l|c|l|l|}
\hline Trial & Treatment & \% Mutant & \% Objective Response (p value within column comparison \\
\hline & & & Wild-type & Mutant \\
\hline CRYSTAL(65) & FOLFIRI vs. FOLFIRI + cetuximab & 35.6 & $\begin{array}{l}43.2 \\
59.3(0.0025)\end{array}$ & $\begin{array}{l}40.2 \\
36.2(0.46)\end{array}$ \\
\hline OPUS(66) & FOLFOX vs. FOLFOX + cetuximab & 42 & $\begin{array}{l}37 \\
61(0.011)\end{array}$ & $\begin{array}{l}49 \\
33(0.106)\end{array}$ \\
\hline EVEREST(67) & $\begin{array}{l}\text { Irinotecan + cetuximab standard dose vs. Irinotecan + } \\
\text { cetuximab dose escalated }\end{array}$ & 36 & $\begin{array}{l}30.4 \\
41.9(0.396)\end{array}$ & 0 \\
0 & $\begin{array}{l}\text { PFS (mo) } \\
12.5 \\
8.6(0.043)\end{array}$ \\
\hline CAIRO 2 ${ }^{*}(68)$ & $\begin{array}{l}\text { CapeOx + bevacizumab vs. CapeOx + bevacizumab + } \\
\text { cetuximab }\end{array}$ & 39 & $\begin{array}{l}\text { PFS (mo) } \\
10.7 \\
10.5(0.10)\end{array}$ & \\
\hline
\end{tabular}

Response rate data not available 